\title{
Smartphone y redes sociales para el desarrollo de competencias mediáticas y digitales en niños y adolescentes: Musical.ly
}

\author{
Ana Pérez-Escoda $\left(^{*}\right)$ y Paloma Contreras Pulido $\left({ }^{* *}\right)$ \\ ${ }^{*}$ ) Universidad Internacional de La Rioja - España $\left(^{* *}\right)$ Universidad de Huelva - España
}

\section{RESUMEN}

La altísima utilización de los smartphones entre la población infantil y adolescente ha propiciado el acceso masivo de este público a las redes sociales, que se han convertido en importantes ámbitos de socialización. Utilizadas como mecanismos de participación, integración e interacción social, niños y adolescentes se han integrado en las redes como escenarios naturales de desarrollo. El objetivo de este trabajo es ofrecer un enfoque pedagógico al estudio de las redes como espacios comunicativos y de desarrollo competencial a través de un estudio de caso de la red Musical.ly. La elección de esta red se justifica por el hecho de que la mayoría de sus 200 millones de usuarios son jóvenes entre 11 y 16 años. La metodología empleada ha sido de corte cualitativo de análisis de contenido basándose en las teorías de la comunicación mediada. A partir del estudio de la estructura mediática de la red, así como de las acciones que posibilita, este trabajo aporta evidencias de la relación que existe entre las acciones que se realizan al utilizar la red social con el desarrollo competencial de la alfabetización mediática y digital, abriendo un relevante campo de investigación que propone las redes sociales como herramientas de aprendizaje.

Palabras Clave: Smartphone, redes sociales, adolescentes, alfabetización mediática, competencia digital.

\section{Smartphone and social networks as enablers of media and digital literacy in children and adolescent: Musical.ly.}

\section{ABSTRACT}

The high penetration of smartphones, especially among children and adolescents has allowed the access to this public to social networks, which have become important areas of socialization for them. Used as mechanisms for participation, integration and social interaction, social networks are used by children and adolescents as natural scenarios in their development. The aim of this work has been to offer a pedagogical approach to the study of social networks, as communicative and competence development spaces, presenting a case study of Musical.ly network. The choice of this social network is justified by the fact that among its 200 million users, the majority are adolescents and children between 11 and 16 years old. The methodology used for the study was a qualitative analysis of content based on the theories of mediated communication. Based on the study of the media structure of the network, as well as the actions it allows, this work provides evidence of the relationship that exists between the actions carried out when using the social network with the competence development of media and digital literacy, opening an interesting field of research that proposes social networks as learning tools.

Keywords: Smartphone, social networks, adolescents, media literacy, digital skills.

\section{Introducción}

El contemporáneo ecosistema tecnológico y cultural en el que vivimos ha favorecido la generación de tres factores que hoy definen y fomentan unas condiciones sociales tan determinantes como inéditas: en primer lugar, la proliferación y digitalización de la información, iniciada a finales del siglo XX junto con el fenómeno de la convergencia mediática, en el que un incipiente Internet comenzó a vertebrar todos los medios conocidos (ㅂiggs,
2017; Jenkins, Ford y Green, 2015); en segundo lugar, y de modo simultáneo, el desarrollo tecnológico, que también inició su despegue en los albores del nuevo siglo con los gigantes de la informática -Microsoft y Apple- y que hoy se desarrolla en dos ámbitos dominantes: gigantescas infraestructuras de redes de banda ancha que soportan toda la información contenida en Internet (5G, IoT, industria 4.0, ciudades inteligentes, etc.) y colosos de la industria informática que brindan dispositivos con funcionalidades más desarrolladas cada año, donde el smartphone se sitúa 
como producto fetiche de una industria creciente e innovadora (CISCO, 2017); y, en tercera pero no menos importante instancia, la aparición de una nueva ciudadanía digital, casi un ecosistema de vida cada vez más dependiente de las funcionalidades digitales que la conexión y los dispositivos permiten (Navarro, 2014). Una ciudadanía basada en una inteligencia cognitiva de relaciones en red, de cultura participativa en la que actitud de compartir, junto con las posibilidades tecnológicas, se aúnan para dar lugar al big data, ingente cantidad de información que todos vuelcan en la red y de la que la inteligencia artificial se nutre directamente para ofrecer unos servicios personalizados, hasta hace poco impensables (Bennett y Segerberg, 2013; CISCO, 2016 García, Del Hoyo y Fernández, 2014). Estos tres fenómenos han hecho que la transformación digital no sea una opción, sino una realidad con peculiaridades significativas y necesidades competenciales mediáticas y digitales específicas que alteran por completo los ámbitos del ser humano tal y como estaban establecidos en la sociedad del siglo XX.

Emerge una nueva economía global donde la información es el único valor de cambio y materia prima de todo el entramado industrial que se genera en este siglo: la revolución de la información (Castells, 2001) y la transformación tecnológica provocan una redistribución de las riquezas y de los valores de consumo (Picketty, 2014). La tecnología permite hablar de dos tendencias: el consumo voraz a través de la tecnología (Sánchez, Fuente y Campos, 2018) y la emergencia de una economía colaborativa, un nuevo modelo de intercambio económico basado en tres aspectos: a) interacción entre el productor y consumidor; b) diálogo continuo, fomentado por las redes sociales; y c) colaboración entre los participantes (crowdsourcing, coworking, bancos de tiempo, etc.) (Cañigueral, 2014). En este contexto cambia de forma paralela el modelo comunicativo propiciado por Internet, que favorecerá una convergencia sin precedentes (Jenkins, Ford y Green, 2015). La red se convierte en el epicentro de la interacción y el paradigma funcionalista de la comunicación lineal, jerarquizada y predeterminada por un emisor y un receptor, se transforma en un esquema abierto, interconectado, descentralizado, dinámico y flexible (Navarro, 2014; Pedrero y Herrera, 2018).

Este cambio de esquema comunicativo se ve reforzado y amplificado por la emergencia y desarrollo de la Web 2.0 que imprime a la comunicación un nuevo y radical enfoque. La web
2.0 transforma la naturaleza del paradigma imperante y cambia los roles de emisores y receptores, favoreciendo la figura del prosumer, usuario que consume y produce conocimiento en redes. Dos visiones interpretan el fenómeno Web 2.0 en este contexto (Lastra, 2016): a) como plataforma interactiva en la que se dibuja una arquitectura participativa cada vez más compleja por las posibilidades que va ofreciendo (software libre, herramientas, almacenamiento en la nube, etc.); y b) como elemento de habilitación social, en el que se genera un nuevo tejido humano basado exclusivamente en las interacciones que ocurren en red.

\section{El smartphone alter ego de las nuevas generaciones}

Cumplidos ya los diez años desde su aparición (el 29 de junio de 2007, Steve Jobs presentaba el primer iPhone), y basando su éxito en la magia de haber conseguido "colocar la potencia de un ordenador en un aparato de bolsillo" y hacer que fuera fácil de utilizar con una pantalla táctil, el smartphone se está convirtiendo en 2018 en el dispositivo más utilizado, a nivel mundial, para acceder a Internet. Pese a un mercado voraz en desarrollo tecnológico, en 2018 el smartphone pugna por convertirse en el dispositivo de uso preferente en manos de los usuarios, sea cual sea su edad. Sin embargo, el gran reto del mercado móvil es que las nuevas generaciones sólo usen este dispositivo. Según el Global Web Index, a finales del 2018 se prevé que el 50\% de los adolescentes-jóvenes sean mobile first, es decir, que usen el smartphone para el $90-100 \%$ de su tiempo en Internet.

La realidad no está lejana a esta previsión, si observamos la madurez del mercado digital se constata que está alcanzando cotas de saturación en muchos países europeos, pero aun así se está produciendo un fenómeno a gran escala en el que correlaciona la omnipresencia del teléfono inteligente con el incremento de la actividad online. Es decir, el smartphone se convierte en el dispositivo por excelencia a través del cual todas las generaciones se conectan a Internet, como puede apreciarse en la Tabla 1. Además, del porcentaje de población que usa smartphone (73\% en 2015, el 96\% se conecta a internet a través de este dispositivo). El crecimiento tanto en uso de este dispositivo como acceso a Internet a través de él experimenta un incremento notable en los últimos años.

Tabla 1. Porcentajes de población en España por edades que usa Internet a diario y que usa smartphone. Fuente: Elaboración propia a partir de The Connected Consumer Survey, 2017.

$\begin{array}{llllllc} & \text { Edad } & 2013 & 2014 & 2015 & 2016 & 2017 \\ & \text { Por debajo 25 } & 89 \% & 87 \% & 88 \% & 92 \% & 100 \% \\ & 25-34 & 83 \% & 84 \% & 92 \% & 96 \% & 98 \% \\ \text { Porcentaje de población que accede a } & 35-44 & 74 \% & 78 \% & 84 \% & 90 \% & 93 \% \\ \text { Internet diariamente } & 45-54 & 54 \% & 65 \% & 70 \% & 76 \% & 85 \% \\ & \text { Más de } 55 & 25 \% & 34 \% & 36 \% & 43 \% & 51 \% \\ & \text { Por debajo } 25 & 85 \% & 98 \% & 96 \% & 98 \% & 100 \% \\ \text { Porcentaje de población que usa } & 25-34 & 85 \% & 95 \% & 98 \% & 99 \% & 98 \% \\ \text { smartphone } & 35-44 & 74 \% & 87 \% & 95 \% & 94 \% & 98 \% \\ & 45-54 & 46 \% & 77 \% & 87 \% & 85 \% & 98 \% \\ & \text { Más de } 55 & 22 \% & 37 \% & 52 \% & 55 \% & 67 \%\end{array}$

Si analizamos los datos concretos en la población por debajo de los 25 años y lo hacemos de modo comparativo con otros países del mundo, los resultados en el caso de España resultan significativos como se aprecia en la Figura 1: 
Figura 1. Porcentajes de crecimiento del uso diario de Internet y de uso de smartphone en diferentes países en población por debajo de los 25 años. Fuente: Elaboración propia a partir de The Connected Consumer Survey, 2017.

$=2017=2016=2015=2014=2013$

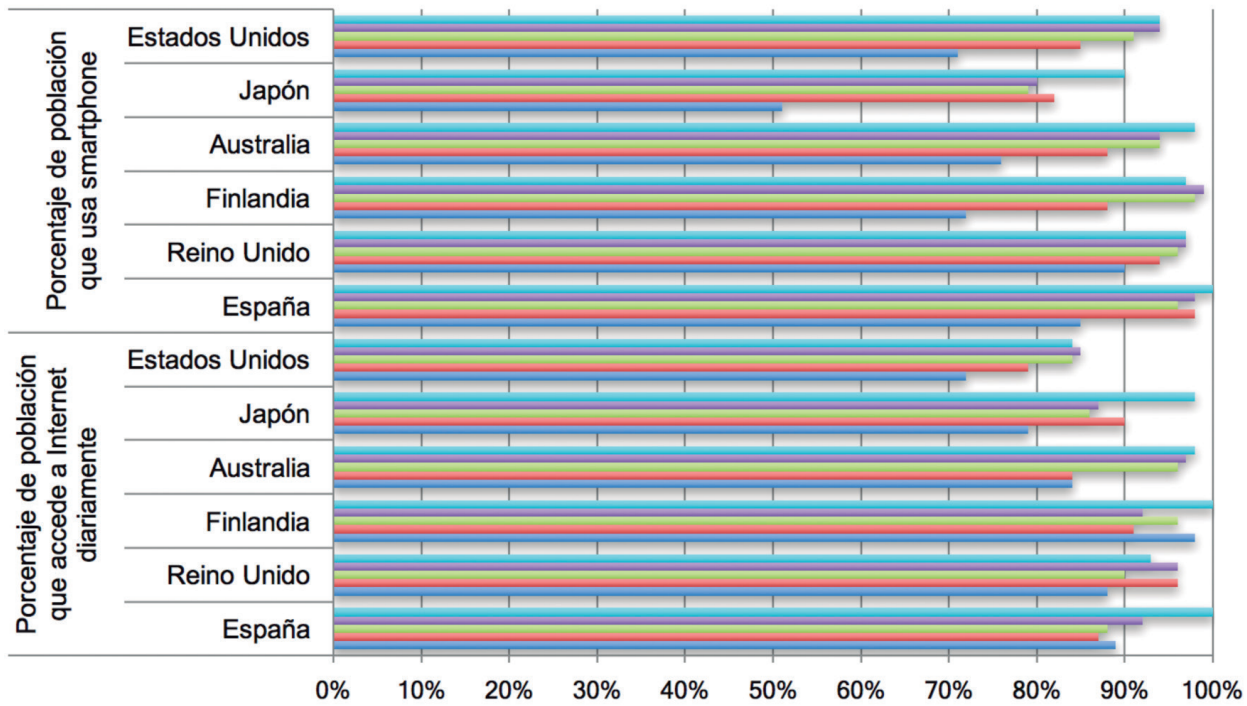

De los países comparados, nuestros jóvenes son los que más acceden a Internet con un porcentaje del 100\% y los que más usan el teléfono inteligente junto con los finlandeses. Los datos sobre estos dispositivos son cada vez más apabullantes y las cifras que alcanzan requieren algo de atención, desde un ámbito de estudio más familiar, el último informe de la consultora Common Sense, publicado en 2017, “Uso de medios y tecnología de los niños entre cero y ocho años", muestra unas conclusiones que permiten obtener una radiografía interesante de cómo los smartphones están cambiando hábitos educativos, familiares y sociológicos. El teléfono inteligente se está convirtiendo en dispositivo casi uni- versal, mientras que en 2011 sólo el 41\% de las familias tenía un dispositivo móvil en el hogar, en 2017, el 95\% tiene uno, es decir, según el estudio, la cantidad de tiempo que los niños pasan frente a las pantallas no ha variado mucho entre 2011 y 2017, lo que sí ha variado sustancialmente es el tiempo dedicado a dispositivos móviles, que desde 2011 se ha triplicado.

Las previsiones de futuro inmediato no auguran que las cifras vayan a bajar en un contexto en el que se prevé que aumente la población que acceda a Internet, el tráfico de datos por persona, las conexiones a banda ancha o la velocidad de los dispositivos móviles conectados a la red como se aprecia en la Figura 2.

Figura 2. Previsión de crecimiento de Internet en los países del Oeste de Europa. Fuente: Elaboración propia a partir de CISCO, 2017.
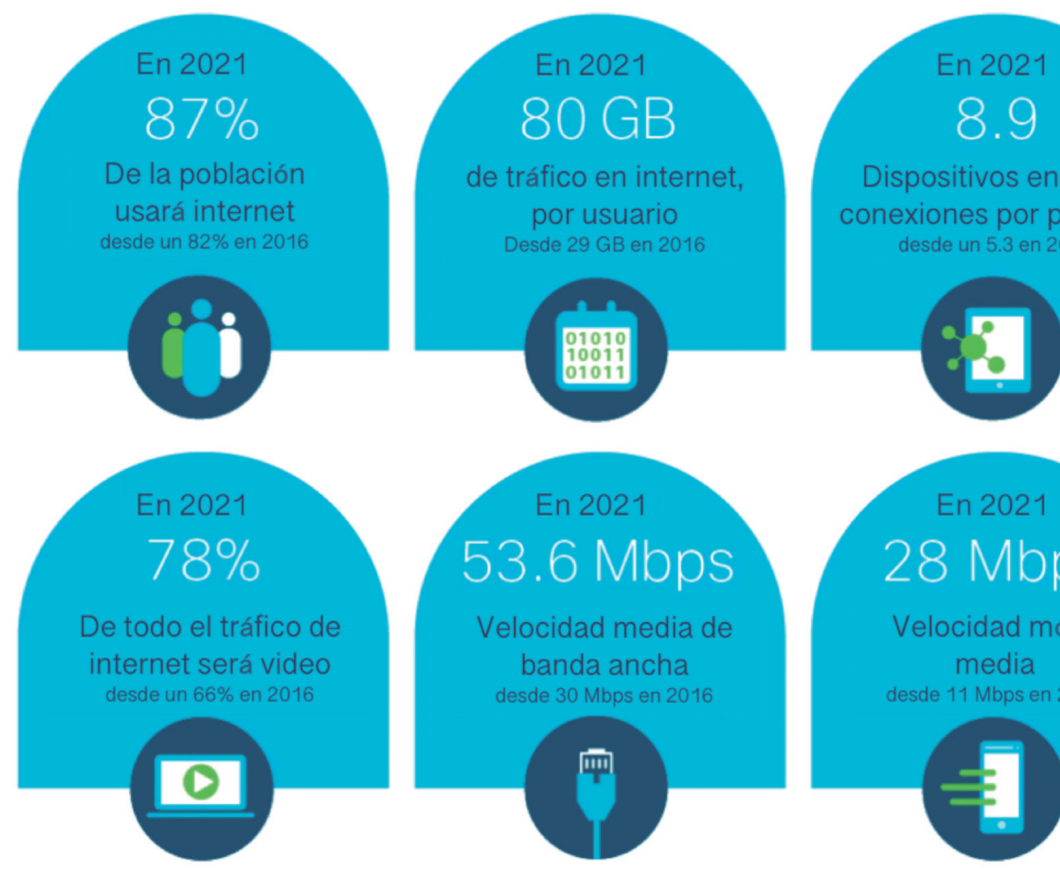

Dispositivos en red y

conexiones por persona

desde un 5.3 en 2016
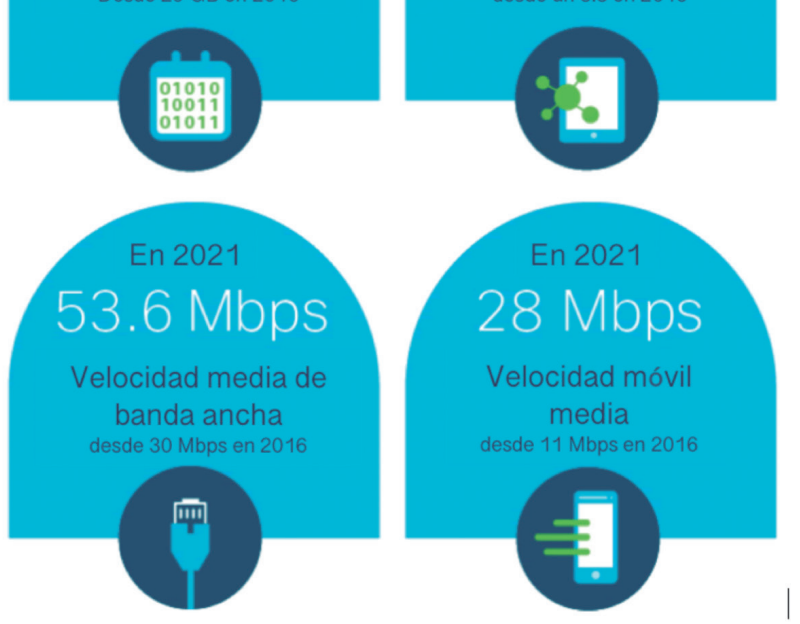

Aula Abierta, volumen 47, nº 3, julio-septiembre, 2018, págs. 281-290 


\section{Cada vez más pronto conectados}

Este marco contextual debe ser interpretado, además, con los datos sobre las edades, cada vez más tempranas, de los usuarios que acceden a un dispositivo conectado, normalmente un smartphone, y que es el enfoque de partida en el que se centra este artículo. Estudios como EU Kid Online de Livingstone, Haddon, Görzig y Ólafsson, (2011), Holloway, Green y Livingstone, (2013), Pérez, Castro y Fandos (2016), Briggs (2017), Vidales y Sábada (2017) demuestran que los niños están conectados a través de diferentes dispositivos proporcionados por sus padres: tabletas, portátiles, pero, sobre todo, smartphones. Según Teuwen, De Groff y Zaman, (2012) el 70\% de los niños prescolares (3-4 años) en Bélgica están conectados a algún dispositivo con Internet; los estudios de Findahl, (2013) dan evidencia de que en Suecia los niños entre 3 y 4 años se conectan a Internet frecuentemente, y el estudio de Chaudron (2015) muestra como en diferentes países de Europa los niños están conectados desde los 0 años de edad hasta los 8 , de un modo familiar y, básicamente, a través de las tabletas. Por otro lado, en el Sur de Corea los niños entre 3 y 9 años se conectan una media de 8-9 horas por semana (Jie, 2012).

También el último informe de Childwise (United Kingdom) publicado en 2017 recoge por la BBC que los niños entre 5 y 16 años pasan más tiempo conectados a Internet (tres horas al día) que viendo la televisión (dos horas al día). Por otro lado, una encuesta llevada a cabo en 2016 por Common Sense a 1.200 familias demostró la incidencia del uso del móvil en las relaciones familiares (Valkenburg y Peter, 2009).

Por tanto, la exposición de las nuevas generaciones al uso de la tecnología conectada deja de ser un hecho anecdótico o puntual para convertirse en un tema central de estudio. Lo que en edades tempranas supone un uso familiar, amigable, dirigido, aunque no por ello inofensivo, cuando llegamos a edades superiores, a partir de los 9 o 10 años, las tabletas son sustituidas por móviles, smartphones conectados a la red. El último estudio del INE, “Menores de Edad y Conectividad Móvil en España muestra que la penetración del móvil entre la población infantil (de 10 a 15 años) ha subido tres puntos en los últimos años, de $67 \%$ en 2015 a casi $70 \%$ en 2017 .

Independientemente de las diferencias generacionales, obvias y necesarias en todas las épocas, las nuevas generaciones tienen una diferenciación mucho mayor, que los aleja cognitivamente más de sus progenitores, son nativos digitales. En este sentido Palfrey y Gasser (2013) argumentaban que la tecnología ha hecho, definitivamente, distintas a estas generaciones por lo que la mayor parte de los aspectos en su vida -interacción social, amistades, actividades sociales- están mediados por tecnologías y no conciben la vida sin ellas. Estudios recientes (García y Fabilia, 2014; García, del Hoyo y Fernández, 2014; De Frutos y Marcos, 2017; Briggs, 2017) profundizan en el calado cuantitativo y cualitativo que el móvil ha producido en la vida de estas generaciones, acentuando su penetración en las diferentes dimensiones vitales:

- Dimensión física o personal, el móvil se ha convertido en una extensión del ser humano hasta el punto de provocar comportamientos adictivos y estados de ansiedad ante la posibilidad de no llevar encima el móvil (García y Fabilia, 2014): la nomofobia (no mobile phone phobia) entendida como un desorden del comportamiento definido como el miedo a quedarse incomunicado tecnológicamente. Este fenómeno ocupa cada vez mayor campo de estudio, demostrando el miedo que tienen las nuevas generaciones de adolescente de salir de casa sin su móvil (Ramos, López y Quiles, 2017). También las evaluaciones internacionales ofrecen datos interesantes en este sentido, el in- forme PISA (OECD, 2017) alerta de que siete de cada diez adolescentes españoles se siente realmente mal si no tiene conexión a Internet.

- Dimensión social, el dispositivo aumenta de modo exponencial las posibilidades de interacción y socialización. Diferentes estudios muestran el fuerte vínculo existente entre el uso del móvil y el aumento de la interacción social (Ling, 2007).

- Dimensión psicológica, en tanto que las redes se convierten en las esferas de mayor influencia para la construcción de identidades en las que el autoconcepto (medido a través de los 'me gustas'), las relaciones interpersonales (medidas a través de los 'seguidores') y el contexto (la propia red social) se convierten en poderosos medios de interacción entre sus iguales.

\section{Las redes sociales}

El estudio de las redes sociales como canales alternativos de diseminación de noticias, construcción de agendas políticas, proliferación de personajes públicos, organizadoras de multitudes, por sus posibilidades comunicativas, ha sido el aspecto más estudiado por la mayoría de autores (Bakshy, Hofman, Mason y Watts, 2011; Bennett y Segerberg, 2013; Peddinti, Ross y Cappos, 2014; Bringgs, 2017). Otra corriente de estudio aborda una perspectiva que encuentra una amplia proliferación por su grado de interés y trascendencia son los estudios como los de Del Rio, Sábada y Bringué (2010), y Catalina, López y García (2014) donde se analizan los riesgos a los que los adolescentes están expuestos con el uso de las redes sociales.

Sin embargo, hay un aspecto relevante que se abre al campo de investigación y que todavía encuentra pocas evidencias académicas, en el que este artículo pretende incidir: el estudio comunicativo de las redes sociales, en tanto que estructuras comunicativas enriquecidas con las posibilidades que Internet y la Web 2.0 propician, y el estudio competencial, es decir, estudiar de modo objetivo, con un análisis estructural y mediático de la red social cuáles son las competencias que su uso desarrolla. Evidentemente, Internet se ha convertido para los menores en un espacio virtual en el que distraerse, interactuar con sus iguales, explorar, conocer cosas nuevas, experimentar nuevas emociones y autoafianzarse como miembros de comunidades activas en las que cobra especial importancia ser aceptados. Pero, ¿qué les aportan?

Estudiar y analizar una red social usada mayoritariamente por niños y adolescentes parece la forma más idónea de encontrar estas evidencias y es la justificación por la que este trabajo se centra en el análisis de la red social del mundo con más usuarios niños y adolescentes: Musical.ly. Musical.ly como red social surge en 2014, de modo fortuito en un tren cuando dos desarrolladores de Silicon Valley, Alex Zhou y Louis Yang, observan a un grupo de chicos grabándose al son de una música de fondo, lo que les anima a diseñar una aplicación con esta funcionalidad. Así, Musical.ly es una aplicación que permite grabar, editar y compartir vídeos musicales (al estilo playback) de hasta 15 segundos. La particularidad de las acciones que permite la red social la hacen idónea para un público que se encuentra entre los 11 y los 16 años, y que conforman el grueso de los 60 millones de usuarios activos que suben al día 13 millones de nuevos clips, del total de 200 millones de usuarios registrados (Smith, 2017).

\section{Nuevas competencias para un contexto}

Resulta de especial interés en este contexto desarrollar un epígrafe específico para hablar de las competencias que de modo 
particular surgen auspiciadas por estos cambios mencionados; aunque no es objetivo de este artículo profundizar, sí cabe esbozar las ideas principales pues sobre dichas competencias se sustenta el análisis de este manuscrito. Siendo de preocupación internacional, la preocupación por el desarrollo de competencias adecuadas a este siglo, surge en 2002 con el Proyecto DeSeCo (OCDE, 2002), como pionero en la definición y profundización de las competencias necesarias para afrontar los retos del siglo XXI. Casi de modo simultáneo, a nivel Europeo se trasladará la preocupación con la Estrategia de Lisboa y las políticas europeas posteriores, ET2010, ET2020, que lo recogerán como punto principal en sus agendas. Sin embargo, no será hasta 2006 cuando se publique la Recomendación del Parlamento Europeo y del Consejo de 18 de diciembre de 2006 sobre competencias clave para el aprendizaje permanente, cuando se encuentre, por primera vez definida, la competencia digital (Pérez-Escoda, 2017). A partir de este momento la competencia digital, desarrollada más adelante por el proyecto DigComp (2011-2017), se incluirá en prácticamente todos los curricula educativos de los países de Europa. De modo paralelo y aunque no incluida en las políticas educativas, la alfabetización mediática se irá desarrollando como necesidad inherente a una sociedad cada vez más visual y mediática (como se verá más adelante con la alta penetración de dispositivos conectados a la red). En este sentido muchos son los estudios que han arrojado sentido y evidencias académicas de la necesidad de estas competencias (Pérez y Varis, 2007; OFCOM, 2009; Ferrés et al., 2011; Aguaded et al., 2011; UNESCO, 2011; Vourikari et al., 2016; Pérez-Escoda, 2017). Para el estudio de las competencias mediáticas y digitales que presenta este artículo, a partir del estudio de caso de la red social Musical.ly se ha elaborado una lista de competencias a partir de los principales trabajos en este campo, organizadas por áreas competenciales:

Tabla 2. Áreas competenciales y subcompetencias de la alfabetización mediática y digital. Fuente: Elaboración propia.

\begin{tabular}{|c|c|c|}
\hline Áreas & & Subcompetencias de cada área competencial \\
\hline $\begin{array}{l}\text { ACCESO: Información y } \\
\text { recepción }\end{array}$ & $\begin{array}{l}1 . \\
2 . \\
3 . \\
4 .\end{array}$ & $\begin{array}{l}\text { Navegación, búsqueda y selección de datos, información y contenido digital } \\
\text { Evaluación de datos, información y contenido digital } \\
\text { Almacenamiento de datos y recuperación de información y contenido digital } \\
\text { Capacidad de selección de información }\end{array}$ \\
\hline $\begin{array}{l}\text { COMUNICACIÓN: } \\
\text { Interacción y uso de } \\
\text { lenguajes }\end{array}$ & $\begin{array}{l}5 . \\
6 . \\
7 . \\
8 .\end{array}$ & $\begin{array}{l}\text { Interacción a través de tecnologías digitales } \\
\text { Cultura participativa y capacidad de influir en opiniones, sentimientos } \\
\text { Colaboración e interacción significativa } \\
\text { Normas de comportamiento online (Netiqueta) }\end{array}$ \\
\hline $\begin{array}{l}\text { CREACIÓN: Contenidos y } \\
\text { Estética }\end{array}$ & $\begin{array}{l}9 . \\
10 . \\
11 . \\
12 .\end{array}$ & $\begin{array}{l}\text { Desarrollo de contenidos digitales } \\
\text { Edición y reelaboración de contenidos digitales } \\
\text { Elaboración de contenidos desde la conciencia de su efecto } \\
\text { Estética de los contenidos que se crean }\end{array}$ \\
\hline $\begin{array}{l}\text { COMPRENSIÓN: } \\
\text { Ideologías y valores }\end{array}$ & $\begin{array}{l}13 . \\
14 . \\
15 . \\
16 .\end{array}$ & $\begin{array}{l}\text { Capacidad para dilucidar por qué gustan unos productos o contenidos, teniendo éxito individu } \\
\text { Protección de los datos personales y privacidad } \\
\text { Capacidad para discernir entre unos contenidos y otros } \\
\text { Gestión de la identidad digital y etiquetado }\end{array}$ \\
\hline $\begin{array}{l}\text { MENEJO: Conocimiento y } \\
\text { uso del medio }\end{array}$ & $\begin{array}{l}17 . \\
18 . \\
19 . \\
20 .\end{array}$ & $\begin{array}{l}\text { Conocimiento y uso de dispositivos y medios } \\
\text { Identificar necesidades y dar respuesta tecnológica } \\
\text { Innovación y creatividad con el uso de las tecnologías digitales } \\
\text { Identificación de carencias en la competencia digital }\end{array}$ \\
\hline
\end{tabular}

\section{Diseño y metodología de la investigación}

En este contexto de uso y penetración del móvil y las redes sociales, se plantea un estudio de caso de la red social Musical. ly, por ser una red social cuyos usuarios son niños y adolescentes -entre 11 y 16 años- con un número de usuarios activos de 60 millones en todo el mundo. El gran alcance de esta peculiar red social entre la población infantil despierta cuanto menos el interés científico y académico, en cuanto a su estructura y sus posibilidades en términos comunicativos y competenciales. El enfoque metodológico utilizado en este estudio de corte cualitativo, se basa en dos enfoques de análisis: a) uno comunicativo, en el que se realizará el análisis de la comunicación en una red social como Musical-ly, a partir de la teoría de los dos escalones de la comunicación de Katz y Lazarsfeld, (Rodrigo, 2001) que se basa en el estudio de la comunicación interpersonal de la comunicación mediada, y en la influencia personal que el líder de opinión (en este caso los musers, perfiles con más seguidores) ejerce sobre sus seguidores (fans), analizando las características de los perfiles más populares sobre el resto de usuarios, a partir de las siguientes variables de estudio: número de fans, número de me gustas, etiquetas de perfil, número de me gustas y de comentarios en los últimos 10 vídeos subidos, así como datos sociodemográficos como la edad y el género. $Y, b$ ), un enfoque competencial que, basado en la teoría de los componentes de la comunicación mediada de Katz, Rice y Acord (2004) y de variables asociadas, ayudará a establecer una base comparativa que correlacione las actividades que se propician en Musical.ly con las competencias mediáticas y digitales establecidas previamente, para demostrar cómo esta red fomenta de modo informal el desarrollo de dichas competencias.

Los datos obtenidos en ambos estudios nos permitirán obtener datos relevantes en clave comunicativa y competencial que ayuden a entender mejor las posibilidades que las redes sociales están ofreciendo a niños y adolescentes. 


\section{Estudio de caso de la red Musical.ly}

En esta primera parte del estudio en el que se pretende analizar la red social desde punto de vista comunicativo, nos basamos en las aportaciones de la teoría de los dos escalones de la comunicación de Katz y Lazarsfeld, (Rodrigo, 2001) en la que primero realizamos un análisis de la estructura mediática de la red, analizando todas las posibilidades de interacción respecto a las siguientes variables: contenidos de perfil, categorización para la publicación de los vídeos, contenidos de los vídeos a los que se puede acceder, posibilidades para la publicación de los vídeos $\mathrm{y}$ tendencias o retos que le red ofrece para animar a sus usuarios a participar en retos colectivos.

Tabla 3. Estructura mediática de la red Musical.ly según las posibilidades de interacción respecto a las variables de estudio. Fuente: Elaboración propia.

Contenidos de perfil

Contenido de los vídeos a los que se puede acceder

Categorización para la publicación de los vídeos

Publicación de contenidos

Reto del momento y Tendencias
Fotografía, Vídeo de biografía, etiquetas (proporcionadas por la red), hashtags, emoticonos, otra cuenta en Instagram, otra cuenta en YouTube y breve descripción biográfica

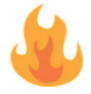

Populares

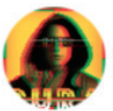

Éxitos
Se trata de los vídeos más populares en el momento en el que el usuario accede a la red

Las mejores canciones: Son los éxitos musicales que se pueden utilizar en los vídeos, hay una doble lista, la global y la de España. $\mathrm{Al}$ acceder a cada canción se muestran vídeos que han usado la canción en cuestión, con la etiqueta de Tendencias

Tabla de clasificación: Se accede a los perfiles de los que han obtenido más corazones "Me gustas" en las siguientes categorías: Populares, España, Global, Comedia, Estilo, Comida, Animales, Talento, Deportes, Vlog, Videojuegos, Arte visual, Música y DIY (Diseño y manualidades).
Los mejores

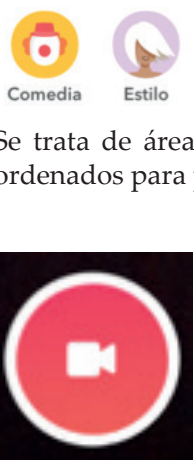

La publicación de contenidos permite una gran variedad de funciones de edición (como se desglosará más adelante) con una duración máxima de 15 segundos por vídeo.

De todas las tendencias que presenta la aplicación (retos que plantea), de modo puntual opta por uno en concreto y lo presenta a modo de anuncio.

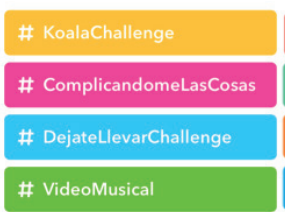

\# LaMúsicaSeApodera
\# TiposDeNovios
\# MeDeclaroAMiCrush
\# BoomFlossChallenge

\begin{tabular}{|l|}
\hline \# NenaMaldición \\
\hline \# ComercialesSinSentido \\
\hline \# RetoDeLaGorra \\
\hline \# DesafioKarmalsAB \\
\hline
\end{tabular}

Todas estas etiquetas, presentadas de este modo en la red, representan diferentes retos que animan a los usuarios a publicar vídeos en diferentes retos

A partir de este análisis de la estructura mediática de la red en las cinco variables estudiadas, se presenta un análisis de los perfiles más famosos. Estos datos nos permiten poner en con- texto el grado de interacción que se concita en la red a partir del número de fans, número de me gustas e interacciones en los últimos 10 vídeos de cada perfil. 
Tabla 4. Análisis de los perfiles más seguidos en la red según el grado de interacción que suscitan. Fuente: Elaboración propia.

\begin{tabular}{lccccc}
\multicolumn{1}{c}{ Nombre } & Edad y sexo & Fans & CT* & Media C** & Media Com. ${ }^{* * *}$ \\
@lorengray & 15 años / M & $23 \mathrm{M}$ & $1,8 \mathrm{~B}$ & 708.440 & 34.212 \\
@babyariel & 16 años / M & $24,4 \mathrm{M}$ & $1,5 \mathrm{~B}$ & 297.105 & 2.138 \\
@jacobsartorius & 14 años / H & $19,4 \mathrm{M}$ & $1,3 \mathrm{~B}$ & 548.290 & 8.516 \\
@lisaandlena & 15 años / M & $26,7 \mathrm{M}$ & $1,9 \mathrm{~B}$ & 1.687 .460 & 36.364 \\
@kristenhancher & 18 años / M & $19,8 \mathrm{M}$ & $1,6 \mathrm{~B}$ & 540.620 & 5.015 \\
@dobretwins & 19 años / H & $10,8 \mathrm{M}$ & $445,6 \mathrm{M}$ & 1.13 .998 & 10.002 \\
@daniellecohn & 13 años / M & $9,97 \mathrm{M}$ & $1,8 \mathrm{~B}$ & 375.480 & 7.664 \\
@rebeccazamolo & 35 años /M & $7,37 \mathrm{M}$ & $1,3 \mathrm{~B}$ & 194.587 & 2.701 \\
@thejamierose & 20 años / M & $4,04 \mathrm{M}$ & $538,9 \mathrm{M}$ & 289.548 & 7.831 \\
@gilmhercroes & 24 años / H & $3,87 \mathrm{M}$ & $314,2 \mathrm{M}$ & 410.190 & 3.623 \\
@justindrewblake & 18 años / H & $3,72 \mathrm{M}$ & $644,2 \mathrm{M}$ & 139.314 & 618
\end{tabular}

${ }^{*}$ Corazones torales del perfil

**Media de corazones obtenidos en los últimos diez vídeos colgados en el perfil

***Media de Comentarios en los 10 últimos vídeos subidos al perfil

Para poder correlacionar las acciones estudiadas con las competencias mediáticas y digitales establecidas y, demostrar así, el potencial de la red como elemento de formación informal, se realizó un estudio profundo de la red por parte de las investigadoras, complementado por entrevistas personales, semiestructuradas a diez adolescentes, usuarias de la red, consistente en enumerar todas las acciones que la red permitía. Así, basándonos en la teoría interpretativa, se fue elaborando un entramado de acciones ordenadas en las cinco variables previamente estudiadas, que ayudaron a establecer la siguiente clasificación de acciones:

\section{A. Relacionadas con el contenido del perfil:}

Editar contenidos personales: nombre, vídeo y breve descripción biográfica

Añadir otras cuentas de Instagram y Youtube

Buscar amigos en Contactos (del móvil), en Facebook

Invitar a amigos mediante mensaje de texto o email

Seguir a la Comunidad en Instagram o Facebook

Comprar camisetas de muser y comprar monedas para comprar regalos

- Obtener ayuda para: hacer un dueto, cargar un vídeo de más de 15 segundos, grabar un vídeo sin mantener presionado el botón, diferenciar entre vídeo privado y público, hacer un vídeo privado, copia de seguridad de mis vídeos privados, borrar vídeos, guardar en la galería

Conectar con otras aplicaciones

Cambiar mi contraseña

Añadir correo secundario para recuperar la cuenta

Ver lista de bloqueados

Configurar la cuenta como privada

Decidir qué notificaciones recibir

B. Relacionadas con el contenido de los vídeos a los que se puede acceder:

- Visitar los vídeos más populares en el momento en el que el usuario accede a la red

- Interactuar y comunicar directamente con esos vídeos a través de "Corazones" y "Comentarios"

- Compartir el vídeo en diferentes aplicaciones: Instagram, Mensaje directo, Whatsapp, Messenger, Facebook, Line, Email, Twitter

- Copiar el enlace del vídeo
- Acceder a la música que utiliza el vídeo para:

- Ver más vídeos que la han utilizado

- Escuchar la canción

- Grabar un vídeo con esa canción

Acceder a vídeos que están retransmitiéndose en directo

C. Relacionadas con la publicación de contenidos:

- Publicar vídeos cómicos accediendo antes a todos los vídeos que se han publicado bajo esta etiqueta

- Publicar vídeos relacionados con el mundo de la belleza y la moda accediendo antes a todos los vídeos que se han publicado bajo esta etiqueta

- Publicar vídeos sobre comida, críticas culinarias, recetas de cocina, etc., accediendo antes a todos los vídeos que se han publicado bajo esta etiqueta

- Publicar vídeos sobre mascotas accediendo antes a todos los vídeos que se han publicado bajo esta etiqueta

- Publicar vídeos sobre talentos específicos: cantar, bailar, interpretar, etc., accediendo antes a todos los vídeos que se han publicado bajo esta etiqueta

- Publicar vídeos sobre momentos de la vida cotidiana (Vlog) accediendo antes a todos los vídeos que se han publicado bajo esta etiqueta

- Publicar vídeos sobre videojuegos accediendo antes a todos los vídeos que se han publicado bajo esta etiqueta

- Publicar vídeos relacionados con artes visuales: pintura, magia, animación, etc., accediendo antes a todos los vídeos que se han publicado bajo esta etiqueta.

- Publicar vídeos componiendo música accediendo antes a todos los vídeos que se han publicado bajo esta etiqueta.

- Publicar vídeos sobre manualidades, diseños, etc. (DIY) accediendo antes a todos los vídeos que se han publicado bajo esta etiqueta

D. Relacionadas con el reto del momento y Tendencias:

Publicar vídeos que respondan a alguna pregunta o reto concreto, accediendo a todos los vídeos que se han subido en ese reto. En todos los retos que se presentan (16 en total) hay uno oficial para que se entienda el reto. Los retos son: \#KoalaChallenge, \#ComplicandomeLasCosas, \#DejateLlevarChallenge, \#VideoMusical, \#LaMusicaSeApodera, \#TiposDeNovios, \#MeDeclaroAMiCrush, \#BoomFlossChallenge, \#RetoNoLove, \#ControlandoPer- 
sonas, \#ComidaFashion, \#TutorialEn30Segundos, \#NenaMaldición, \#ComercialesSinSentido, \#RetoDeLaGorra, \#DesafioKarmalsAB
El análisis realizado respecto a todas estas acciones que permite la red social resulta fundamental para establecer relaciones y conexiones con las diferentes subcompetencias predeterminadas de la alfabetización mediática y digital, evidenciando el papel de la red social como garante de estas competencias constitutivas de la alfabetización que niños y adolescentes necesitan.

Tabla 5. Relación establecida entre áreas competenciales de la alfabetización mediática y digital y acciones en Musical.ly. Fuente: Elaboración propia.

\begin{tabular}{|c|c|}
\hline Áreas & Acciones que se engloban en cada área competencial \\
\hline $\begin{array}{l}\text { MANEJO: Conocimiento } \\
\text { y uso del medio }\end{array}$ & 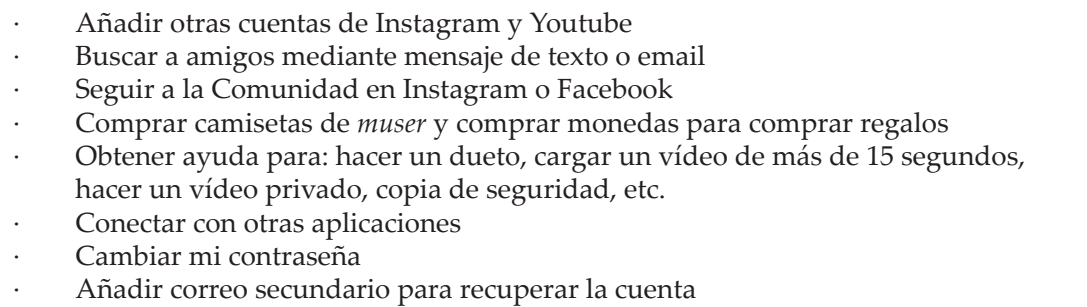 \\
\hline $\begin{array}{l}\text { CREACIÓN: Contenidos } \\
\text { y estética }\end{array}$ & $\begin{array}{l}\text { Ver lista de bloqueados } \\
\text { Configurar la cuenta como privada }\end{array}$ \\
\hline $\begin{array}{l}\text { COMUNICACIÓN: } \\
\text { Interacción y uso de } \\
\text { lenguajes }\end{array}$ & $\begin{array}{l}\text { Editar contenidos personales: nombre, vídeo y breve descripción biográfica } \\
\text { Invitar a amigos mediante mensaje de texto o email } \\
\text { Seguir a la Comunidad en Instagram o Facebook } \\
\text {. Comprar camisetas de muser y comprar monedas para comprar regalos } \\
\text { Obtener ayuda para: hacer un dueto, cargar un vídeo de más de } 15 \text { segundos, } \\
\text { grabar un vídeo sin mantener presionado el botón, diferenciar entre vídeo privado } \\
\text { y público, hacer un vídeo privado, copia de seguridad de mis vídeos privados, } \\
\text { borrar vídeos, guardar en la galería } \\
\text { Conectar con otras aplicaciones } \\
\text { Interactuar y comunicar directa con esos vídeos a través de "Corazones" y } \\
\text { comentarios" }\end{array}$ \\
\hline $\begin{array}{l}\text { CREACIÓN: Contenidos } \\
\text { y estética }\end{array}$ & $\begin{array}{l}\text { Compartir el vídeo en diferentes aplicaciones: Instagram, Mensaje directo, } \\
\text { Whatsapp, Messenger, Facebook, Line, Email, Twitter } \\
\text {. Copiar el enlace del vídeo } \\
\text {. } \\
\text { Acceder a la música que utiliza el vídeo para: } \\
\text {. } \quad \text { Escr más vídeos que la han utilizado } \\
\text {. } \quad \text { Grabar un vídeo con esa canción } \\
\text { Acceder a vídeos que están retransmitiéndose en directo }\end{array}$ \\
\hline $\begin{array}{l}\text { MANEJO: Conocimiento } \\
\text { y uso del medio }\end{array}$ & $\begin{array}{l}\text { Crear y publicar vídeos sobre los diferentes tópicos ofrecidos } \\
\text { Crear y publicar vídeos sobre algún reto concreto }\end{array}$ \\
\hline $\begin{array}{l}\text { COMPRENSIÓN: } \\
\text { Ideologías y valores }\end{array}$ & $\begin{array}{l}\text {. } \quad \text { Cambiar mi contraseña } \\
\text {. } \quad \text { Añadir correo secundario para recuperar la cuenta } \\
\text { Ver lista de bloqueados }\end{array}$ \\
\hline $\begin{array}{l}\text { MANEJO: Conocimiento } \\
\text { y uso del medio }\end{array}$ & $\begin{array}{l}\text { Configurar la cuenta como privada } \\
\text { Decidir qué notificaciones recibir } \\
\text { Visitar los vídeos más populares en el momento en el que el usuario accede a la } \\
\text { red }\end{array}$ \\
\hline
\end{tabular}

Entiendo que los participantes son los adolescentes, así lo dicen, pero no sabemos nada de sus características sociodemográficas, no lo describe, solo esto: semiestructuradas a diez adolescentes, usuarias de la red, consistente en enumerar todas las acciones que la red permitía.

\section{Discusión y conclusiones}

El estudio de las redes sociales se ha abordado en los últimos años desde diferentes perspectivas: desde la participación activa de los jóvenes (García-Galera, del-Hoyo-Hurtado y Fernández-Muñoz, 2014), desde perspectivas afectivo-familiares y de relaciones (Takeuchi, 2011; Padilla, Rodríguez, Álvarez, Torres, Suárez y Rodrigo, 2015; Schrock, 2016; Vidales y Sábada, 2017), desde las experiencias negativas y percepción de riesgo (De-Fru- tos y Marcos, 2017; Peddinti, Ross y Cappos, 2014; Radesky, Schumacher y Zuckerman, 2015), desde la relación entre jóvenes y tecnología (Lenhart, Smith, Anderson, Duggan y Perrin, 2015), sin embargo, se echan en falta enfoques más pedagógicos que aborden el estudio de las redes sociales como espacios comunicativos y de desarrollo competencial. Es en este punto donde el artículo que aquí se presenta pretende abrir un nuevo campo de investigación, aportando este estudio de caso. Es cierto que el trabajo presentado adolece de carencias y limitaciones, metodológicas y de alcance suficiente, que deben ser mejorados en estudios posteriores, pero el trabajo presenta una primera aproximación a este enfoque.

Se concluye del trabajo realizado que, de modo ineludible, las redes sociales están presentes en la vida de niños y adolescentes, lo cual induce a recapacitar sobre cómo se están cons- 
truyendo las lógicas comunicativas, que asientan en los jóvenes unas lógicas sociales distintas y que los convierte en usuarios críticos, activos y con un grado de responsabilidad asumida de forma espontánea en entornos donde sus progenitores y educadores no intervienen. Es cierto que el estudio llevado a cabo no permite llegar a conclusiones de gran alcance, aunque sí permite realizar unas primeras aproximaciones sobre los beneficios que el uso de estas redes imprimen en el desarrollo competencial de los adolescentes debido a las funcionalidades que presentan. Estas posibilidades se relacionan directamente con la alfabetización mediática y digital: evaluación y búsqueda de contenidos digitales, creación y edición de contenidos digitales, desarrollo de habilidades estéticas y comunicativas en entornos virtuales, es decir, nuevos lenguajes y códigos comunicativos. Después de analizar la estructura mediática de Musical.ly y categorizar las acciones que permite en función de unas variables elegidas para el estudio, y relacionarlas con competencias de la alfabetización mediática y digital, no parece descabellado afirmar que el uso de estas redes propicia competencias mediáticas y digitales.

Por lo tanto, sería relevante, de cara al futuro, centrar el ámbito de estudio de las redes sociales hacia enfoques constructivistas y pedagógicos en los que se analicen las virtudes y los beneficios que están suponiendo para jóvenes y adultos. Las abrumadoras cifras de usuarios tan jóvenes hacen que deba replantearse el modo en el que estas redes se usan, normalmente en entornos informales, para poder incluirlas en las realidades de aula, aprovechando el gran potencial comunicativo y competencial que en estudios como este se evidencian.

\section{Financiación}

Este trabajo está avalado por el Proyecto I+D+I, titulado “Competencias mediáticas de la ciudadanía en medios digitales emergentes (smartphones y tablets): prácticas innovadoras y estrategias educomunicativas en contextos múltiples" con clave EDU2015-64015-C3-1-R (MINECO/FEDER), financiado por el Fondo Europeo de Desarrollo Regional (FEDER) y Ministerio de Economía y Competitividad de España.

\section{Referencias bibliográficas}

Aguaded, J. I., Ferrés i Prats, J., Cruz Díaz, M. R., Pérez Rodríguez, M. A., Sánchez Carrero, J. y Delgado Ponce, Á. (2011). El grado de competencia mediática en la ciudadanía andaluza. Huelva: Grupo Comunicar Ediciones/Grupo de Investigación Ágora, Universidad de Huelva.

Bakshy, E., Hofman, J.M., Mason, W.A., y Watts, D.J. (2011). Everyone's an influencer: Quantifying influence on twitter. Proceedings of the fourth ACM International Conference on Web Search and Data Mining (pp. 65-74). ACM. https://doi. org/10.1145/1935826.1935845

Bennett, W.L. y Segerberg, A. (2013). The logic of connective action: Digital media and the personalization of contentious politics. Massachusetts: Cambridge University Press. (https://goo.gl/ NxxV1Z).

Briggs J. (2017). Young people and participation in Europe. London: Palgrave Macmillan. https://doi.org/10.1057/978-1-137-31385-0

Buckingham, D. y Willet, R. (2013) (Ed.). Digital generations. Children, young people and New Media. London: Routledge.

Catalina García, B., López de Ayala, M.C. y García Jiménez, A. (2014). Los riesgos de los adolescentes en Internet: los menores como actores y víctimas de los peligros de Internet. Revista Latina de Comunicación Social, 69, 462-485. DOI: 10.4185/ RLCS-2014-1020
Cañigueral Bagó, A. (2014). Vivir mejor con menos. Consumo colaborativo. Barcelona: Liberdúplex. Bajo Licencia Creative Commons.

Chaudron, S. (2015). Young Children (0-8) and Digital Technology: A qualitative exploratory study across seven countries. Luxembourg: Publications Office of the European Union. https:// doi.org/10.2788/00749

CISCO. (2016). Global Mobile Data Traffic forecast, 2015-2020. Recuperado de: http://www.cisco.com/c/en/us/solutions/collateral/service-provider/visual-networking-index-vni/vni-forecast-qa.pdf

CISCO (2017). Cisco visual Networking Index: Forecast and Methodology, 2016-2021. Recuperado de: https://www.cisco.com/c/ en/us/solutions/collateral/service-provider/visual-networking-index-vni/complete-white-paper-c11-481360.html

Common Sense Media. (2017). Media Use by kids age Zero to Eight. Recuperado de: https://www.commonsensemedia.org/sites/ default/files/uploads/pdfs/2017-zero-to-eight-not-infographic-final.pdf

De-Frutos, B. y Marcos, M. (2017). Disociación entre las experiencias negativas y la percepción de riesgo de las redes sociales en adolescentes. El Profesional de la Información, 26(1), 88-96. https://doi.org/10.3145/epi.2017.ene.09

Ferrés, J., García, A., Aguaded, J. I., Fernández, J., Figueras, M., Blanes, M., et al. (2011). Competencia mediática. Investigación sobre el grado de competencia de la ciudadanía en España. Madrid: Ministerio de Educación.

García-Galera, M., del-Hoyo-Hurtado, M., y Fernández-Muñoz, C. (2014). Jóvenes comprometidos en la Red: El papel de las redes sociales en la participación social activa. [Engaged youth in Internet. The role of social networks in social active participation]. Comunicar, 22(43), 35-43. https://doi.org/10.3916/ C43-2014-03

García-Martínez, V. y Fabilia Echauri, A.M. (2014). Nomofilia vs. Nomofobia, irrupción del teléfono móvil en las dimensiones de vida de los jóvenes. Un tema pendiente para estudios en comunicación. Razón y Palabra [en línea] 2014. http://www. redalyc.org/articulo.oa?id=199530728048

Jenkins, H., Ford, S., y Green, J. (2015). Cultura transmedia: la creación de contenido y valor en una cultura en Red. Barcelona: Gedisa.

(https://goo.gl/XRYKeB).

Katz, J., Rice, R., y Acord, S. (2004). Personal Mediated Communication and the Concept of Community in Theory and Practice. En P.J. Kalbfleisch, (Ed.), Communication and Community, Communication Yearbook 28 (pp. 315-371). Mahwah, NJ: Erlbaum. (http://goo.gl/2Z22Ci)

Lastra, A. (2016) El poder del prosumidor. Identificación de necesidades y repercussion en la producción audiovisual transmedia. Icono 14, Vol. 14(1), 71-94. https://doi.org/10.7195/ri14. v14i1.902

Lenhart, A., Smith, A., Anderson, M., Duggan, M., y Perrin, A. (2015). Teens, technology and friendships. Pew Research Center. (https://goo.gl/PHq81a).

Ling, R. (2007). Children, youth and mobile communications. Journal of Children and Media (1) 1, 60-67.

Navarro, L.R. (2014). Entre esferas públicas y ciudadanía. Las teorías de Arendt, Habermas y Mouffe aplicadas a la comunicación para el cambio social. Barcelona: UOC. (https://goo.gl/KYfekS).

OCDE-DeSeCo (2002). Definition and selection of competencies: Theoretical and conceptual foundations. Recuperado el 10/01/2018 de: http://www.portalstat.admin.ch/deseco/index.htm.

OECD (2017). PISA 2015 Results (Volume III): Students'Wellbeing. Paris: OECD Publishing. http://dx.doi.org/10.1787/9789264273856-en 
Ofcom. (2009). Audit of learning-related media literacy policy development. Recuperado de http://stakeholders.ofcom.org.uk/ binaries/research/media-literacy/Education_Policy_Audit_ for_1.pdf

Padilla, S., Rodríguez, E., Álvarez, M., Torres, A., Suárez, A., y Rodrigo, M.J. (2015). La influencia del escenario educativo familiar en el uso de Internet en los niños de primaria y secundaria. Infancia y Aprendizaje, 38(2), 402-434. https://doi.org /10.1080/02103702.2015.1016749

Palfrey, J. G., y Gasser, U. (2013). Born digital: Understanding the first generation of digital natives. Basic Books.

Peddinti, S.T., Ross, K.W., y Cappos, J. (2014). On the Internet, nobody knows you're a dog: A Twitter case study of anonymity in social networks. Proceedings of the Second ACM Conference on Online Social Networks (pp. 83-94). Nueva York: ACM. (https://goo.gl/pAgVTL).

Pedrero-Esteban, L.M. y Herrera-Damas, S. (2017). La notificación push como estrategia informativa de la radio en el entorno digital. El profesional de la información, Vol. 26(6), 1100-1107. DOI: https://doi.org/10.3145/epi.2017.nov.09

Pérez-Escoda, A. (2017). Alfabetización mediática, TIC y competencias digitales. Barcelona: UOC.

Pérez-Escoda, A., Castro-Zubizarreta, A. y Fandos, M. (2016). Digital Skills in the Z Generation: Key Questions for a Curricular Introduction in Primary School. [La competencia digital de la Generación Z: claves para su introducción curricular en la Educación Primaria]. Comunicar, 49, 71-79. https://doi. org/10.3916/C49-2016-07

Pérez-Tornero, J.M., Celop, P. y Varis, T. (2007). Study on the Current Trends and Approaches to Media Literacy in Europe. Recuperado de http://ec.europa.eu/culture/library/studies/ literacy-trends-report_en.pdf

Radesky, J.S., Schumacher, J., y Zuckerman, B. (2015). Mobile and interactive media use by young children: The good, the bad, and the unknown. Pediatrics, 135(1), 1-3. DOI: 10.1542/ peds.2014-2251
Rodrigo Alsina, M. (2001). Teorías de la Comunicación. Ámbitos, métodos y perspectivas. Zaragoza: INO Reproducciones, S.L.

Sánchez-Bayón, A., Fuente, C. y Campos, G. (2018). Plan de acción frente al consumismo global de la Nueva Economía: Revelaciones sobre economía, empresa y consumo en el siglo XXI. Revista Empresa y Humanismo, Vol. XXI, 1, 69-93. DOI: https://doi.org/10.15581/015.XXI.1.69-93

Takeuchi, L.M. (2011). Families matter: Designing media for a digital age. New York: The Joan Ganz Cooney Center at Sesame Workshop. (https://goo.gl/on63qU).

Rodríguez-Canfranc, P. y Castillo-Holgado, A. (2017). Sociedad Digital en España 2017. Fundación Telefónica. Madrid: Ariel.

Ramos-Soler, I., López-Sánchez, C., y Quiles-Soler, M. (2017). Adaptación y validación de la escala de nomofobia de Yildrim y Correia en estudiantes españoles de la Educación Secundaria. Salud y drogas, 17 (2), 201-213. Recuperado de: http://ojs.haaj.org/index.php/haaj/article/view/332

Schrock, A.R. (2016). Exploring the Relationship between Mobile Facebook and Social Capital: What Is the "Mobile Difference" for Parents of Young Children? Social Media E Society, 2(3). https://doi.org/10.1177/2056305116662163

Smith, C. (2017). 22 Musical.ly Statistics and Facts. Recuperado de Business Statistics en enero de 2018: https://expandedramblings.com/index.php/musically-statistics/

Picketty, T. (2014). El capital en el siglo XXI. Madrid: Fondo de Cultura Económica de España.

Vidales-Bolaños, M., y Sábada-Chalezquer, C. (2017). Adolescentes conectados: La medición del impacto del móvil en las relaciones sociales desde el capital social. Comunicar, 25(53), 19-28. https://doi.org/10.3916/C53-2017-02

Vuorikari, R., Punie, Y., Carretero, S., y Brande, L. Van den. (2016). DigComp 2.0: The digital competence framework for citizens. Update Phase 1: The Conceptual Reference Model. Luxembourg: European Comission. 